\title{
Stem cell pathologies and neurological disease
}

\author{
Dennis A Steindler ${ }^{1}$, Michael S Okun² and Björn Scheffler ${ }^{3}$ \\ ${ }^{1}$ Department of Neurosurgery, College of Medicine of the University of Florida, Gainesville, FL, USA; \\ ${ }^{2} U F$ Center for Movement Disorders and Neurorestoration, Departments of Neurology and Neurosurgery, \\ University of Florida, Gainesville, FL, USA and ${ }^{3}$ The Institute of Reconstructive Neurobiology, University \\ of Bonn/Life and Brain, Bonn, Germany
}

\begin{abstract}
The presence of stem and progenitor cells in the adult human brain suggests a putative and persistent role in reparative behaviors following neurological injury and neurological disease. Too few stem/progenitor cells (as in the case of Parkinson's disease) or too many of these cells (as in the case of Huntington's disease and glioma) could contribute to and even signal brain pathology. We address here critical issues faced by the field of stem cell biology and regenerative medicine, arguing from well-documented as well as speculative perspectives for a potential role for stem cells in the pathology of many human neurological diseases. Although stem cell responses may result in regenerative failure, in many cases they may help in the establishment or re-establishment of a functional neural circuitry (eg, after stroke). Therefore, we would argue that stem cells have a crucial-either positive or negative-role in the pathology of many neurological diseases.
\end{abstract}

Modern Pathology (2012) 25, 157-162; doi:10.1038/modpathol.2011.165; published online 4 November 2011

Keywords: brain tumor; neurodegenerative disease; regeneration failure; stem cell

\section{Somatic stem and progenitor cells should be ready to repair tissue, but their potency is vulnerable}

The field of stem cell biology has provided a great deal of discovery as well as reversal of developmental biology dogma since its birth in the 1950s with radiation biology studies. The revival of the field following the seminal discoveries of Stevens, Evans, Martin, Reynolds and Weiss, and Thomson (see ref. 1) has provided the stimulus for explosive growth. There is likewise growing interest in translating new discoveries, especially in embryonic and induced pluripotent stem cell (iPSC) biology, ${ }^{2,3}$ into regenerative medicine (as in bone marrow transplantation for cancer, and recently, bone marrow or even peripheral blood hematopoietic stem cells for autoimmune disorders ${ }^{4}$ ). There are anticipated nearfuture new treatments and possibly cures for a variety of diseases and injuries (eg, Geron Corporation embryonic stem cell trial for acute spinal cord injury) dependent on advances in our understanding

Correspondence: Professor DA Steindler, PhD, Joseph J. Bagnor/ Shands Professor of Medical Research, Department of Neurosurgery, College of Medicine, McKnight Brain Institute of The University of Florida, 100 S. Newell Drive, Gainesville, FL 32610, USA.

E-mail: Steindler@neurosurgery.ufl.edu

Received 8 June 2011; revised 5 September 2011; accepted 06 September 2011; published online 4 November 2011 of the nature of these potent cells. ${ }^{5}$ Most human organ systems contain self-renewing cells that under many circumstances can succeed at tissue regeneration as well as restoration of functional integrity following indigenous stem and progenitor cell response to an injury/ disease. These systems can accomplish this task with impressive regenerative competence. By definition, potent stem/progenitor cells should innately respond to injury or disease with enhanced proliferation, and with homing to compromised tissue and organ sites. The cells utilize well-characterized chemokine-based biotropism (eg, SDF-1/cxcr4), where they attempt replacement and repair. When successful, cells may be continuously replaced and with reintegration not being noticed and surprisingly there seems to be little to no 'downtime' for the organ. On the other hand, it must be carefully considered that this continual reactive cell replacement by stem/progenitor cells within mature tissues may provide a conducive setting for overgrowth, ie, neoplastic events, when cells are susceptible to oncogenic transformation.

Neurodegenerative diseases and stroke as examples of failed regeneration resulting from stem cell pathologies

The brain, for a long time assumed not to undergo much replacement and restoration following 
injury/disease, is now generally believed to be a regenerative organ. The brain possesses its own unique populations of stem and progenitor cells that can respond to cell loss with targeted emigration of neuronal and glial progenitor cells from confined niches such as the subventricular zone. It is therefore tempting to hypothesize that some neurodegenerative diseases reflect 'stem cell pathologies', where stem/progenitor cell populations not only contribute to the disease state directly (via providing abnormal cellular replacements during normal neuropoiesis) but also fail to provide the requisite cellular replacements for those lost as a result of the disease state. In Huntington's disease (HD), for example, the subventricular zone exhibits altered neural stem/progenitor cell behavior indicative of reactive failed neurogenic attempts, ${ }^{6-8}$ including a potential increase in numbers of stem cells. ${ }^{8}$ The same principle holds true for stroke, although the attempt may be considered efficient and also commendable, as cells home to infarcted cortical sites and differentiate. However, $80 \%$ of these cells die within weeks of the stroke, due to an environment that is presumably not conducive for successful functional integration. ${ }^{9}$ Furthermore, there are cycling 'satellite' cells within the adult substantia nigra $^{10}$ that may be willing to provide replacement cells in diseases such as Parkinson's (PD) (eg, see Figure 1), but they may need coaxing via the discovery of neurogenic factors that support their proliferation, differentiation and survival.

\section{Mechanisms of disease conveyance to and from stem cells}

These cells also seem to fail at reactive neurogenesis in vivo possibly due to genetic, molecular and cellular consequences of PD or possibly from being overwhelmed by massive need beyond their numbers. In PD, recent studies have shown that certain disease-associated proteins, eg, alpha synuclein, may exhibit infectious behaviors, ${ }^{11-16}$ with neuronal and exosomal ${ }^{17}$ transcellular conductance of the infectious protein such that transplanted fetal neural precursor cells contract PD-associated synucleinopathies. This would represent another form of stem cell pathology, as stem/progenitor cells within fetal neural transplants would be at-risk for infection, and once infected may not be able to respond to injury and tissue rebuilding cues as their normal counterparts do. Likewise, compromised endogenous stem/progenitor cells reside within the mid- and forebrains in PD, and because of both the abnormal genomes they possess or as a result of exposure to infectious proteins, including synuclein, these cells may be rendered chronically compromised during attempted tissue repair. This stem cell pathology appears to involve exosomal transmission ${ }^{17}$ of infectious proteins as well as potentially altered intracellular protein degradation events, ${ }^{18}$ which together contribute to the disease state. Such a combination of abnormal proteins and cell behaviors offers insight into the potential mechanism of disease transmission that may go beyond stem cell pathologies in PD and also include $\mathrm{HD}^{19}$ (where connections from existing pathological forebrain circuitry have been imputed to cause degeneration within non-HD donor-derived fetal tissue transplants in the HD striatum) as well as other neurodegenerative diseases.

\section{Cancerous stem cells contribute to diverse tissue pathologies}

Even though stem cells offer hope for new cellular and molecular treatments for human disease, they may also serve as perpetrators, and there has been a current focus on so-called 'cancer stem cells'. Whether or not a normal stem or progenitor cell can 'go bad' and become a so-called cancer stem cell, at the absolute least, the study of stem cell biology does provide potentially valuable insights into the nature and phenotype of cancer and tumor-initiating cells. It certainly is also possible that a cancerous somatic cell acquires stem cell-like attributes and behaviors during the course of neoplastic disease. As mentioned above, when stem cells respond to routine illness/injury-induced cell loss, tissue repair is accomplished and integration occurs appropriately with little or no 'downtime' for the organ. When stem cells potentially respond to injury/ disease too exuberantly and in association with the activation of oncogenes and the inactivation of cancer-suppressing genes, then oncogenic transformation and hyperplasia/neoplasia could ensue. Such a process may take its toll on a tissue or on a organ, and 'cancers' could conceivably come and go, depending on the physiological state of the individual. The strength of the immune system and each individual's potential to incorporate altered cells into an established cell network without serious consequences may impact the outcome. In other words, altered or abnormal 'poiesis' may be occurring continuously as a normal human biological process, and the products of this process may be vulnerable to be attacked and even eliminated by a brisk and appropriate immune response. Some individuals however may be overwhelmed (eg, via a compromised state of health), and the tendency toward neoplasia may 'win'. Cancer can thus be viewed in a larger context as a stem or progenitor cell attempting to fulfill its prearranged role with the physiology of the human, whether related to normal cell turnover in the brain or to reactive neurogenesis, if it might occur following an injury or disease. There are examples of the reactive glial cells in an attempted repair of the brain, ${ }^{20}$ whereby a subset of reactive astrocytes in response to injury upregulate the same growth-associated genes and proteins, eg, extracellular matrix and associated molecules, 


\section{The complex world of brain stem cell pathologies}

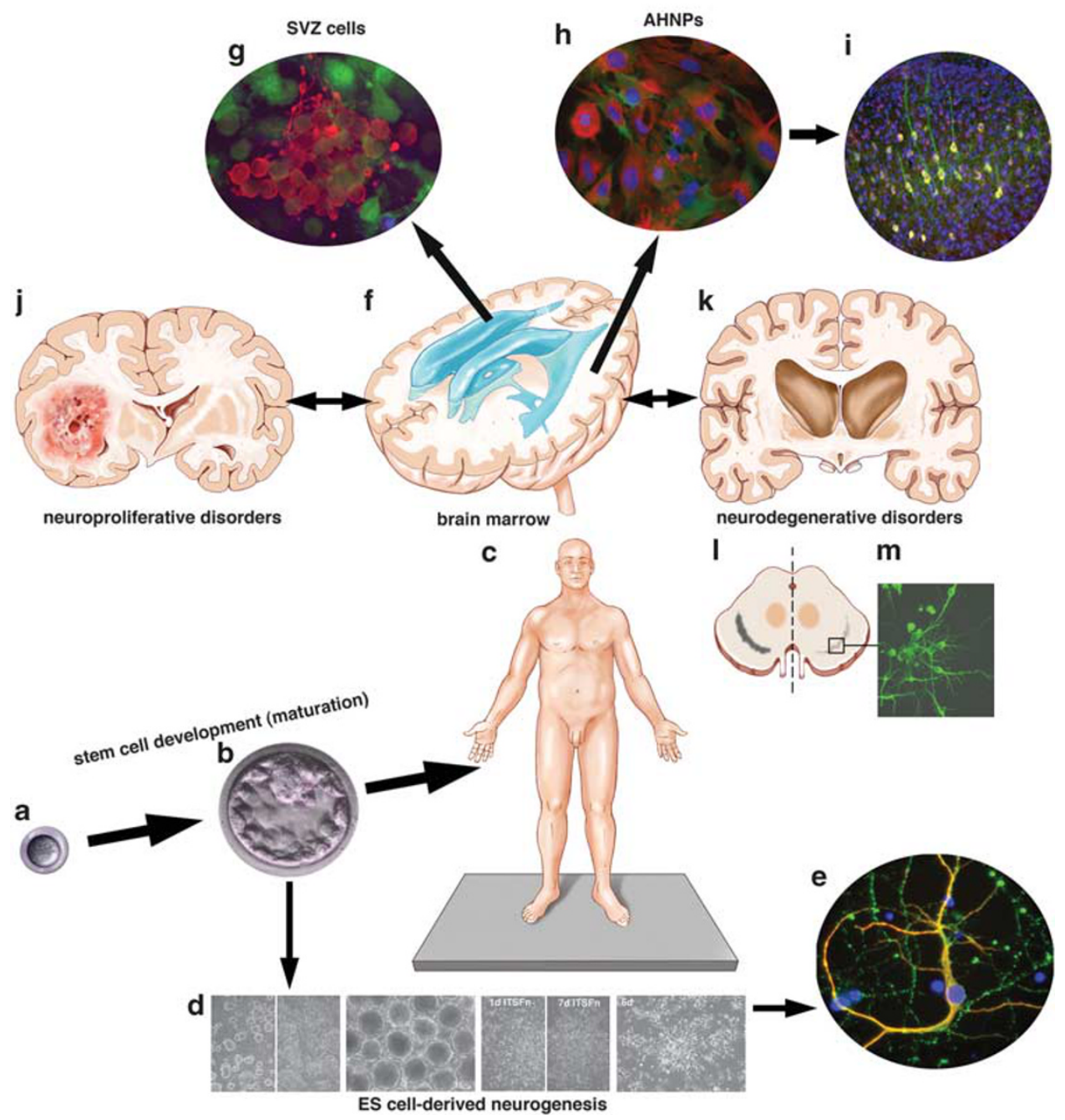

Figure 1 The complex world of stem cell pathologies. Tissue-generating/repairing stem cells function from the beginning to the end of life (a-c): from the zygote (a), to the blastocyst (b), to a fully mature human being (c) where somatic or tissue-specific stem and progenitor cells reside in many, if not all, tissues and organs. Their lack or abundance may indicate stem cell pathologies. Although source and nature of most neurodegenerative and neuroproliferative disorders remain currently unknown, the study of stem cells in all stages of development may open new avenues for diagnosis and treatment of cancer and other diseases through clues from regenerative medicine. Embryonic stem cells (d) derived from the blastocyst inner cell mass (b) can give rise to all tissues and organs, including neural precursor cells that participate in embryonic and adult neurogenesis and generate different types of differentiated neurons (e) and glia. Neural stem and progenitor cells reside in the adult human brain in the periventricular subventricular zone (SVZ, and f) and the hippocampus, and participate in persistent neurogenesis (neuropoiesis, ${ }^{1}$ and g) that can be reactive cell genesis following neurodegenerative disease ${ }^{5-8}$ injury or stroke. ${ }^{9}$ Progenitor cells can also be found in adult human brain cortical gray matter (so-called 'Adult Human Neural Progenitor Cells', or 'AHNPs', h, and see ref. 37) that can be massively propagated in vitro and seemingly amenable to reparative attempts in vivo, as grafting these cells into the cerebrum of adult SCID mice results in the generation of differentiated cells, including neurons (i). Neuropoiesis in the human brain can also lead to too much growth (j), as seen in our originally described cancer stem cell population involved in gliomagenesis. ${ }^{38}$ Attempted but failed regeneration of neurons from stem and progenitor cell populations in neurodegenerative diseases including Huntington's disease (k) suggests that reactive neurogenesis could be enhanced and exploited with molecular factors and drugs discovered from new bioassays and high-throughput screening approaches. This is exemplified in vitro from pilot studies in which we isolate cells from postmortem specimens of the substantia nigra in another neurodegenerative disease, Parkinson's disease (PD; 1 ), and even though stem/progenitor cells in this nucleus do not successfully thwart the loss of dopaminergic neurons in this disease in vivo, they may be amenable to the generation of neurons (eg, beta III tubulin-positive) and dopamine-like neurons (tyrosine hydroxylase-positive) under the proper growth conditions in vitro (m), and then used for in vitro bioassaying for drug screening, or compared with cells generated from new reprogramming ${ }^{24,25}$ methods (ie, induced pluripotent stem cells, (iPSCs), as described for PD and other neurodegenerative diseases ${ }^{39,40}$ ). Ex vivo study and manipulation of these neural precursor cells may provide insights into tapping these cells for self-repair in PD and other neurodegenerative diseases. All of these examples illustrate the potential roles for stem cells in the etiology of disease (eg, neurodegenerative or neoplastic), as well as during attempted repair and regeneration of tissues and organs following injury or disease. The neostriatum atrophies significantly through progressive stages of $\mathrm{HD}(\mathbf{k})$, with loss of both neurons and glia over time; ${ }^{41}$ it is clear that reactive neurogenesis with stem cell enhanced proliferation occurs at the expense of neuronal progenitor cells, however, ${ }^{42}$ that therefore fails to hold the basal ganglia and cortical cell loss at bay without a necessary compensatory reactive neurogenesis. Figures 'd and e' are from the study by Goetz et al (PNAS 2006; 103:11063-8); Figure ' $\mathrm{g}$ ' is from the study by Scheffler et al (PNAS 2005; 102:9353-8) and, Figures ' $\mathbf{h}$ and $\mathbf{i}$ ' are from the study by Walton et al. ${ }^{37}$ 
which also are expressed by glial cells both during development and cancer. ${ }^{21}$ The cell is simply generating tissue, and only when affected by genetic alteration, miscellaneous environmental 'hits,' and by oncogenic transformation, does the cell overgenerate and yield a cell lineage diversity that results in neoplasm. There is data to suggest that the state of a neural stem/progenitor cell involved in generating new neurons is vulnerable to transformation because of unique cell cycle activities as well as aneuploidy $^{22}$ that could lead to neoplasia. Be it either the generation of new cells for normal replacement following age-related or other cell loss or to attempt the replacement and repair following more extensive degeneration following injury or disease, cell genesis is certainly not without risk from a mitotically active, hence, vulnerable population of cells that are capable of giving rise to phenotypically diverse lineages and overgrowth. Such a neoplasm might fail to integrate within the tissue mass and eventually destruction and even metastasis may follow.

In all, would it be therefore not reasonable to suggest that many diseases reflect 'stem cell pathologies' in the sense that stem cells, either overwhelmed or transformed, really represent another underlying cause that also contributes to disease?

\section{Conclusion}

\section{Biomarkers for and Treatments of Neural Stem Cell Pathologies}

This proposed role for stem cells in cancer and other diseases, although avant-garde, has appeal based on recent scientific data from the regenerative medicine field. Tissues and organs may accommodate for alterations of the stem cell pool at early disease stages; eg, greater than half of the substantia nigra neurons in PD are lost before clinically relevant symptoms. The same principle seems to be followed in neuroproliferative disease, where the brain may have undergone huge structural changes without any clinically relevant symptomatology. Thus, the onset of symptoms when we detect them in clinical medicine may correlate with a more advanced stage of disease. At these advanced stages, it becomes difficult to demonstrate with certainty that the absence of or the genetic alteration of rare populations of stem cells (even under physiological conditions) may have contributed to the genesis of pathology.

Stem cell numbers are increased in many neoplastic disorders (eg, brain, breast, bone, and colon cancer and melanoma). These stem cells can induce the formation of cancer-like lesions when engrafted in animal models of disease. Lessons from the field of hematopoietic research have demonstrated that these cancerous stem cells, as alluded to above, may not necessarily represent the founder population, but rather, and at least in some cases, reflect the result of disease progression following the acquisition of stem cell characteristics. ${ }^{23}$ It thus may be more practical for diagnosis and treatment of neoplastic disorders, if stem cells are not viewed as a group of distinct physical entities, but are seen as a description of a conditional functional activity state that may occur for any cell during disease. This view would agree with seminal studies by the Yamanaka and Thomson groups demonstrating that any cell could acquire stem cell characteristics. ${ }^{24,25}$ Fibroblasts can be, for example, reprogrammed through retroviral transduction to become pluripotent embryonic stem cell-like, and adult neural cells can be coaxed or dedifferentiated into more primitive precursors. These precursors can repopulate potentially vast territories of the CNS, and certain genetic events can drive committed blood precursor cells to become normal transdifferentiated, (eg, blood to brain), precursor cells. These cells can integrate in even well-established CNS circuitries. ${ }^{1}$ Finally, the phenomenon of stem/progenitor cell fusion ${ }^{26,27}$ must also be considered both as a basis and also as a treatment for disease. ${ }^{28}$ In the liver, for example, the regeneration of the organ takes place following disease/injury and may result from fusion of bone marrow stem cells to polyploidal hepatic oval cells. This fusion could contribute to genetic instability and hyperplasia, and this could provide a basis for non-neural cells associated with glioblastoma or mesenchymal stem cells that are rumored to occur in breast cancer. ${ }^{29}$

In conclusion, the presence of too many (as in potentially certain stages of HD and definitely with glioma) or too few (PD and any other neurodegenerative disease where aging and infectious proteins are just two of many risk factors), stem cells could indicate pathology. Further consideration is necessary by both researchers and clinicians to demonstrate the source and nature of stem cell pathologies, especially with regard to translating insights from stem cell biology to regenerative medicine. We should however entertain the possible notion that many neurological diseases reflect stem cell pathologies. That is, a stem cell's job is to rebuild diseased or injured tissue, and in light of strong evidence for a well-positioned pool of neurogenic and potentially reparative stem/progenitor cells in the human brain throughout life, their inability to replace lost cells and restore function undoubtedly can be considered as a pathology, a stem cell pathology. There is now growing evidence that stem cells attempt regeneration in the injured and diseased CNS, with even the glial scar ${ }^{30}$ being composed in part of astrocytic cells whose main job is not aimed at inhibiting neurite growth through their expressions of extracellular matrix and other repulsive molecules, but rather, these cells are attempting reactive neurogenesis where proliferation, fate choice and differentiation is the major goal with their creation of a neurogenic molecular environment that secondarily does not support the growth of new neurites. Though looking like regenerative failure, in fact adult neural 
stem/progenitor cells actually achieve a great deal toward the establishment or re-establishment of functional neural circuitry, with a stem cell pathology being their inability to complete the job due to age-related constraints or toxic disease-associated environments.

Equally daunting is the potential stem cell pathological state of creating too much tissue, as proposed for cancer stem cells involved in the gliomas. As exosomes from pathological stem cells, eg, glioblastoma cancer stem cells, may express unique proteins, mRNAs and miRNAs, ${ }^{31}$ that represent powerful new biomarkers present in serum, disease progression and treatment-associated remissions, could be monitored using peripheral blood. Some of these same pathological stem cell markers may turn out to also be strategic targets of therapeutic approaches that both modify undesired stem cell behaviors as well as counter cell-to-cell spread of disease-inducing factors. A very recent paper has shown that an abnormal gene expression in some cells differentiated from iPSCs can induce a T-cell-dependent immune response in syngeneic recipients, ${ }^{32}$ and thus iPSC pathologies should also be considered before embarking on clinical applications of this exciting but nevertheless rather new cellular therapeutic technology. Another recent study has shown that prostate cancer stem cells naturally express the four iPSC reprogramming factors, ${ }^{33}$ adding further credence to a notion that stem cells are highly potent and yet vulnerable cells that offer both hope, as potential tissue-repairing therapeutics, as well as concern for their potential primary role in the etiology of a variety of neurological and other disease states. It is no surprise then that the multilineage cell and genetic diversity, well-recognized as a hallmark of the cancers, is associated with both cancer stem-like cells, such as those seen in prostate tumor-initiating cells as well as iPSCs that express the Oct-3/4, Klf4, Sox2 and c-Myc reprogramming and oncogenic genes. ${ }^{34-36}$ If disease states themselves or successive cell divisions result in genetic aberrations of these and associated stemness pathways, additional levels of daughter heterogeneity result and generate cells that may fall short of disease repair and also contribute to disease. Trying to harness desired normal stem/progenitor cells for repair that must also dedifferentiate to a highly potent state before contributing to extensive proliferation and then differentiation with multilineage replacement cell diversity following induction or grafting within a neurological disease state is a challenge, but with emerging technologies to help control potent cell behaviors, certainly not without possibility for repair and restoration of lost circuitry.

\section{Acknowledgements}

DAS is supported by the NIH/NINDS R01 grant NS055165 and the Maren, Thompson and
McKinney Regenerative Medicine funds; MSO is supported by grants from the National Parkinson Foundation, the NIH/NINDS, the Parkinson Alliance and the Michael J. Fox Foundation. BS is supported by the Lichtenberg program of the VW foundation. We thank Dr A. Katrin Goetz and Dr Noah Walton for their help in generating some of the data in Figure 1 and David Peace for help with making the Figure.

\section{Disclosure/conflict of interest}

The authors declare no conflict of interest.

\section{References}

1 Steindler DA, Pincus DW. Stem cells and neuropoiesis in the adult human brain. Lancet 2002;359:1047-1054.

2 Yamanaka S. Strategies and new developments in the generation of patient- specific pleuripotent stem cells. Cell Stem Cell 2007;1:39-49.

3 Cohen DE, Melton D. Turning straw into gold: directing cell fate for regenerative medicine. Nat Rev Genet 2011;12:243-252.

4 Burt RK, Loh Y, Cohen B, et al. Autologous nonmyeloablative haemopoietic stem cell transplantation in relapsing-remitting multiple sclerosis: a phase I/II study. Lancet Neurol 2009;8:244-253.

5 Conti L, Cattaneo E. Neural stem cell systems: physiological players or in vitro entities. Nat Rev Neurosci 2010;11:176-187.

6 Curtis MA, Faull RL, Eriksson PS. The effect of neurodegenerative diseases on the subventricular zone. Nat Rev Neurosci 2007a;8:712-723.

7 Curtis MA, Eriksson PS, Faull RL. Progenitor cells and adult neurogenesis in neurodegenerative diseases and injuries of the basal ganglia. Clin Exp Pharmacol Physiol 2007b;34:528-532.

8 Curtis MA, Penney EB, Pearson AG, et al. Increased cell proliferation and neurogenesis in the adult human Huntington's disease brain. Proc Natl Acad Sci USA 2003;100:9023-9027.

9 Arvidsson A, Collin T, Kirik D, et al. Neuronal replacement from endogenous precursors in the adult brain after stroke. Nat Med 2002;8:963-970.

10 Lie DC, Dziewczapolski G, Willhoite AR, et al. The adult substantia nigra contains progenitor cells with neurogenic potential. J Neurosci 2002;22:6639-6649.

11 Kordower JH, Chu Y, Hauser RA, et al. Lewy body-like pathology in long-term embryonic nigral transplants in Parkinson's disease. Nat Med 2008;14:504-506.

$12 \mathrm{Li} \mathrm{JY}$, Englund E, Holton JL, et al. Lewy bodies in grafted neurons in subjects with Parkinson's disease suggest host-to-graft disease propagation. Nat Med. 2008;14:501-503.

13 Mendez I, Viñuela A, Astradsson A, et al. Dopamine neurons implanted into people with Parkinson's disease survive without pathology for 14 years. Nat Med. 2008;14:507-509.

14 Olanow CW, Prusiner SB. Is Parkinson's disease a prion disorder? Proc Natl Acad Sci USA 2009;106: 12571-12572.

15 Angot E, Steiner JA, Hansen C, et al. Are synucleinopathies prion-like disorders? Lancet Neurol 2010;9: 1128-1138. 
16 Hansen C, Angot E, Bergström AL, et al. $\alpha$-Synuclein propagates from mouse brain to grafted dopaminergic neurons and seeds aggregation in cultured human cells. J Clin Invest 2011;121:715-725

17 Emmanouilidou E, Melachroinou K, Roumeliotis T, et al. Cell-produced alpha-synuclein is secreted in a calcium-dependent manner by exosomes and impacts neuronal survival. J Neurosci 2010;30:6838-6851.

18 Alvarez-Erviti L, Seow Y, Schapira AH, et al. Lysosomal dysfunction increases exosome-mediated alphasynuclein release and transmission. Neurobiol Dis 2011;42:360-367.

19 Cicchetti F, Saporta S, Hauser RA, et al. Neural transplants in patients with Huntington's disease undergo disease-like neuronal degeneration. Proc Natl Acad Sci USA 2009;106:12483-12488.

20 Laywell ED, Bartsch U, Dorries U, et al. Enhanced expression of the developmentally regulated extracellular matrix molecule tenascin following adult brain injury. Proc Natl Acad Sci USA 1992;89:2634-2638.

21 Silver D, Steindler DA. Common astrocytic programs during brain development, injury and cancer. Trends Neurosci 2009;32:303-311.

22 Walton NM, Kobeissy F, Scheffler B, et al. Gliotypic neural stem cells transiently adopt tumorigenic properties during normal differentiation. Stem Cells 2009; 27:280-289.

23 Passegue E, Jamieson CH, Ailles LE, et al. Normal and leukemic hematopoiesis: are leukemias a stem cell disorder or a reacquisition of stem cell characteristics? Proc Natl Acad Sci USA 2003;100:S1:11842-S1:11849.

24 Takahashi K, Tanabe K, Ohnuki M, et al. Induction of pluripotent stem cells from adult human fibroblasts by defined factors. Cell 2007;131:861-872.

$25 \mathrm{Yu}$ J, Vodyanik MA, Smuga-Otto K, et al. Induced pluripotent stem cell lines derived from human somatic cells. Science 2007;318:1917-1920.

26 Terada N, Hamazaki T, Oka M, et al. Bone marrow cells adopt the phenotype of other cells by spontaneous cell fusion. Nature 2002;416:542-545.

27 Ying QL, Nichols J, Evans EP, et al. Changing potency by spontaneous fusion. Nature 2002;416:545-548.

28 Chen KA, Cruz PE, Lanuto DJ, et al. Cell fusion for gene delivery to SCA1 affected Purkinje neurons. Mol Cell Neurosci 2011;47:61-70.

29 Karnoub AE, Dash AB, Vo AP, et al. Mesenchymal stem cells within tumour stroma promote breast cancer metastasis. Nature 2007;449:557-556.
30 Buffo A, Rite I, Tripathi P, et al. Origin and progeny of reactive gliosis: A sourece of multipotent cells in the injured brain. Proc Natl Acad Sci USA 2008;105: 3581-3586.

31 Skog J, Würdinger T, van Rijn S, et al. Glioblastoma microvesicles transport RNA and proteins that promote tumour growth and provide diagnostic biomarkers. Nat Cell Biol 2008;10:1470-1476.

32 Zhao T, Zhang Z-N, Rong Z, et al. Immunogenecity of induced pluripotent stem cells. Nature 2011;474:212-215.

33 Bae KM, Su Z, Wang S, et al. Expression of pluripotent stem cell reprogramming factors by prostate tumorinitiating cells. J Urol 2010;183:2045-2053.

$34 \mathrm{Hu}$ BY, Weick JP, Yu J, et al. Neural differentiation of human induced pluripotent stem cells follows developmental principles but with variable potency. Proc Natl Acad Sci USA 2010;107:4335-4340.

35 Hussein SM, Batada NN, Vuoristo S, et al. Copy number variation and selection during reprogramming to pluripotency. Nature 2011;471:68-62.

36 Gore A, Li Z, Fung HL, et al. Somatic coding mutations in human induced pluripotent stem cells. Nature 2011;471:63-67.

37 Walton NM, Sutter BM, Chen HX, et al. Derivation and large-scale expansion of multipotent astroglial neural progenitors from adult human brain. Development 2006;133:3671-3681.

38 Ignatova TN, Kukekov VG, Laywell ED, et al. Human cortical glial tumors contain neural stem-like cells expressing astroglial and neuronal markers in vitro. Glia 2002;39:193-206.

39 Park IH, Arora N, Huo $\mathrm{H}$, et al. Disease-specific induced pluripotent stem cells. Cell 2008;134: 877-886.

40 Soldner F, Hockemeyer D, Beard C, et al. Parkinson's disease patient-derived induced pluripotent stem cells free of viral reprogramming factors. Cell 2009; 136:964-977.

41 Thomas LB, Gates D, O'Brien TF, et al. DNA end labeling (TUNEL) in Huntington's disease and other neuropathological conditions. Exp Neurol 1995;133: 265-272.

42 Kandasamy M, Couillard-Despres S, Raber KA, et al. Stem cell quiescence in the hippocampal neurogenic niche is associated with elevated transforming growth factor-beta signaling in an animal model of Huntington disease. J Neuropathol Exp Neurol. 2010; 69:717-728. 